\title{
Amplification of realistic Schrödinger-cat-state-like states by homodyne heralding
}

Laghaout, Amine; Neergaard-Nielsen, Jonas S.; Rigas, loannes; Kragh, Christian; Tipsmark, Anders; Andersen, Ulrik L.

Published in:

Physical Review A

Link to article, DOI:

10.1103/PhysRevA.87.043826

Publication date:

2013

Document Version

Publisher's PDF, also known as Version of record

Link back to DTU Orbit

Citation (APA):

Laghaout, A., Neergaard-Nielsen, J. S., Rigas, I., Kragh, C., Tipsmark, A., \& Andersen, U. L. (2013).

Amplification of realistic Schrödinger-cat-state-like states by homodyne heralding. Physical Review A, 87(4), 043826. https://doi.org/10.1103/PhysRevA.87.043826

\section{General rights}

Copyright and moral rights for the publications made accessible in the public portal are retained by the authors and/or other copyright owners and it is a condition of accessing publications that users recognise and abide by the legal requirements associated with these rights.

- Users may download and print one copy of any publication from the public portal for the purpose of private study or research.

- You may not further distribute the material or use it for any profit-making activity or commercial gain

- You may freely distribute the URL identifying the publication in the public portal

If you believe that this document breaches copyright please contact us providing details, and we will remove access to the work immediately and investigate your claim 


\title{
Amplification of realistic Schrödinger-cat-state-like states by homodyne heralding
}

\author{
Amine Laghaout, ${ }^{1}$ Jonas S. Neergaard-Nielsen, ${ }^{1}$ Ioannes Rigas, ${ }^{2}$ Christian Kragh, ${ }^{1}$ Anders Tipsmark, ${ }^{1}$ and Ulrik L. Andersen ${ }^{1,2}$ \\ ${ }^{1}$ Department of Physics, Technical University of Denmark, Building 309, 2800 Lyngby, Denmark \\ ${ }^{2}$ Quantum Information Theory Group, Institut für Theoretische Physik I, and Max-Planck Research Group, Institute of Optics, \\ Information and Photonics, Universität Erlangen-Nürnberg, Staudtstraße 7/B2, 91058 Erlangen, Germany
}

(Received 31 January 2013; published 18 April 2013)

\begin{abstract}
We present a scheme for the amplification of Schrödinger cat states that collapses two smaller states onto their constructive interference via a homodyne projection. We analyze the performance of the amplification in terms of fidelity and success rate when the input consists of either exact coherent state superpositions or of photon-subtracted squeezed vacua. The impact of imprecise homodyne detection and of impure squeezing is quantified. We also assess the scalability of iterated amplifications.
\end{abstract}

DOI: 10.1103/PhysRevA.87.043826

PACS number(s): 42.50.Dv, 42.50.Ex

\section{INTRODUCTION}

Coherent state superpositions, or optical Schrödinger cat states, are widely recognized as promising resources in quantum information [1-5], quantum metrology [6-8], and fundamental tests [9-12]. In the near-orthogonal basis of coherent states $\langle\gamma \mid-\gamma\rangle=e^{-2 \gamma^{2}}$, two particular instances for these states are

$$
\left|\kappa_{ \pm}(\gamma)\right\rangle=\frac{1}{\sqrt{2 \pm 2 e^{-2|\gamma|^{2}}}}(|\gamma\rangle \pm|-\gamma\rangle),
$$

where the sign $( \pm)$ of the superposition refers to the even and odd cat state, respectively. These states exhibit quasiprobability distributions in phase space which are distinctly nonclassical. This makes them all the more challenging to generate deterministically as that would require strong Kerr-type nonlinearities [13-15]. One has then to resort to heralding techniques which, though probabilistic, need only linear optics and projective measurements [16]. These state-engineering schemes are nonetheless approximative and present a limitation in the fidelity they produce with ideal cat states. Photon subtraction of the squeezed vacuum, for example, is a well-established method to generate approximations of small-amplitude cat states, sometimes colloquially referred to as Schrödinger kittens [17-21]. Even in the best experimental conditions, the fidelity between the photonsubtracted squeezed vacuum (PSSV) and an actual odd cat state $\left|\kappa_{-}(\gamma)\right\rangle$ degrades markedly for $\gamma \geqslant 1.2$ [22]. Yet, for these states to be reliable resources in quantum computation, their fidelity with cat states at least as large as $\gamma=1.2$ needs to be maintained at near-unit fidelity $[3,23]$.

Single-photon subtraction is only one example of several measurement-induced schemes which have been proposed to generate small-amplitude cat states [20,24-29]. However, none of these schemes can produce arbitrarily large cat states in a single run. Ways to get around this issue have been devised using the recursive amplification of small, approximate cat states [30,31]. For example, it was suggested in [32] that interference among a supply of delocalized single photons followed by homodyne heralding would generate large entangled cat states. These proposals have in common that they rely on the coherent mixing of two small cat states, whereupon a projective measurement collapses one of the two outputs onto a constructive interference of the inputs-hence the amplification. Here, we shall pursue the same idea but make use solely of homodyne heralding for its relative simplicity and high quantum efficiency. We also demonstrate that the acceptance window of homodyne heralding can be widened to increase the success rate of the amplification while at the same time maintaining a satisfactory fidelity at the output.

The outline of this article is as follows. In Sec. II we review the generation of odd small-amplitude cat states from the squeezed vacuum. The output is compared to the ideal odd cat state and the effects of impure squeezing are illustrated. In Sec. III we present the amplification scheme in the case of ideal input cat states and model the effect of a wide homodyning window. Section IV then considers the amplification of the more realistic PSSV. The impact of both impure squeezing and wide postselection is illustrated. In Sec. V, we return to the idealized case of ideal homodyning and pure squeezing to consider how our scheme scales with iterated amplifications.

\section{APPROXIMATION OF SMALL ODD CAT STATES}

In this section, we shall briefly review the generation of odd small-amplitude cat states from the photon subtraction of the squeezed vacuum and analyze its performance in the face of impure squeezing. The basic setup is depicted in Fig. 1. The original proposal of Dakna et al. [17] required that the photon subtraction be performed by photon-number-resolving detectors. However, as is done in most practical schemes $[18,19,21]$, we shall assume that the postselection is a binary detection of either zero photons or at least one photon, as would be allowed for by an avalanche photodiode (APD). The modeling of such an "on-off" post-selection operation is presented in Appendix A 3.

Quantum inefficiencies and dark counts are two nuisances inherent to photodetection which should be reckoned with. Whereas the former merely affects the success probability of the scheme, the latter contaminates the output with a squeezedvacuum component which weakens the nonclassicality of the output. An equally detrimental effect is the impurity of the squeezed vacuum. We shall not treat quantum inefficiencies and dark counts here as they have already been covered in [16] in the context of cat-state amplification. We will, however, look at the fidelity response to the amplitude and impurity of squeezing. 


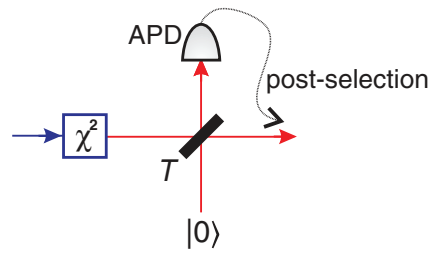

FIG. 1. (Color online) Setup for the generation of approximate small-amplitude odd cat states. A squeezed vacuum, represented here by the pumping of a $\chi^{2}$ nonlinear medium, is partially reflected onto an "on-off" photon detector such as an avalanche photodiode (APD). Upon the reflection of at least one photon from the squeezed vacuum, a state that likens the squeezed single photon is prepared in the limit of unit transmission $T \rightarrow 1$.

\section{A. Fidelity of the output for pure squeezing}

Let us assume from now on that the squeezed vacuum is pure and antisqueezed in the $x$ quadrature. That is, if we denote the squeezing relative to the shot-noise variance by $\xi$ (in $\mathrm{dB}$ ), then $\xi_{x}=-\xi_{p}>0$, where the subscript labels the measured quadrature. ${ }^{1}$ The fidelity of the PSSV state with an ideal cat state $\left|\kappa_{-}(\alpha)\right\rangle$ then depends on the squeezing $\xi$ and the transmission $T$ of the subtraction beam splitter. ${ }^{2}$ The effect of these parameters is illustrated in Fig. 2 where we can see that the fidelity is optimized for low squeezing and near-unit beam-splitter transmission $T \rightarrow 1$. For any given input squeezing, there corresponds a finite amplitude $\alpha$ of the target cat state with which the output has a maximized fidelity. For example, a squeezing of, say, $3 \mathrm{~dB}$ is optimal for producing an approximation to a cat state of size $\alpha=1.0$. A complementary investigation of PSSV states that looked at nonclassicality as the main figure of merit (as opposed to fidelity) is given by Kim et al. in [33].

In order to assess the performance of the PSSV generation, it is most instructive to restrict ourselves to the maximal fidelity achievable with any target cat state of size $\alpha$. This fidelity optimum is traced by the ridge of Fig. 2 (top) and its $\alpha$ dependence is reproduced in Fig. 2 (bottom) along with the corresponding success rate. For example, if one wants to produce an approximate odd cat state of size $\alpha=1.5$, the required squeezing should be $\xi \approx 5.2 \mathrm{~dB}$ for a fidelity of at most $95.4 \%$ and a success probability of $0.4 \%$.

As far as the success probability is concerned, it can be increased at the expense of fidelity by increasing the incidence of photons on the APD via stronger squeezing or weaker beamsplitter transmission.

\footnotetext{
${ }^{1}$ For simplicity, we shall refer to both squeezing and antisqueezing as squeezing $\xi$. The difference between the two is made only by the sign of $\xi$.

${ }^{2}$ The transmission of the subtraction beam splitter does not affect just the success probability of the scheme, but also the fidelity of the output state. This is because we are using an on-off postselection where we assume that by setting $T \rightarrow 1$, only one photon will makes its way to the APD. A lower transmission would furthermore inflict higher loss of the initial squeezed state.
}
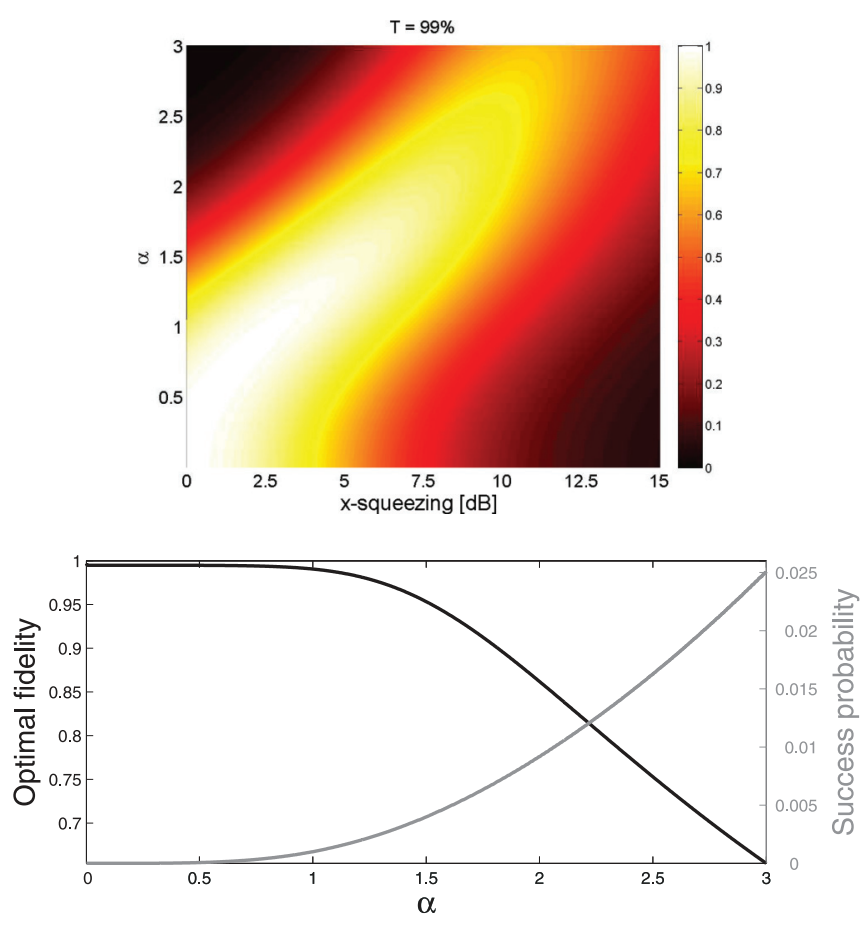

FIG. 2. (Color online) Top: Contour plot of the fidelity of the PSSV state with $\left|\kappa_{-}(\alpha)\right\rangle$ given an input squeezing $\xi_{x}$ between 0 and $15 \mathrm{~dB}$. Bottom: Maximum achievable fidelity of the PSSV with $\left|\kappa_{-}(\alpha)\right\rangle$ (black) and corresponding success probability as a function of $\alpha$ (gray). In both cases, the transmission of the subtraction beam splitter is $99 \%$.

\section{B. Impact of impure squeezing}

In any real-world experiment, noise will inevitably inflate the variance of the squeezed vacuum in either quadrature. This impurity has been explained as stemming from losses or from multimode parametric down-conversion [34] whereby the photons triggering the postselection belong to a different spatial or frequency mode than the heralded state. Impure squeezing may originate in the down-converter itself or, more generally, at any point in the setup where vacuum contamination or modal mismatch could take place, including at the detectors (e.g., via quantum inefficiencies). Regardless of its root causes, we shall wrap these impurities into a single parameter $\epsilon$ relating the squeezing $\xi$ in $\mathrm{dB}$ of the $x$ and $p$ quadratures,

$$
\xi_{x}=-\epsilon \xi_{p},
$$

whereby pure squeezing corresponds to $\epsilon=1$. As derived in Appendix A 1, the purity of the squeezed vacuum is given by

$$
\mathcal{P}=10^{-(1 / 20)(1-\epsilon) \xi_{p}} \text {. }
$$

Note that the Heisenberg uncertainty relation imposes that $\epsilon \geqslant 1$.

The impact of impurity on fidelity is plotted in Fig. 3 where we set the squeezing at $-3.0 \mathrm{~dB}$ and adjust the antisqueezing according to four different settings of purity. The immediate observation is that a decrease of $10 \%$ in purity, from $100 \%$ to $90 \%$, corresponding to an increase of antisqueezing to $+3.9 \mathrm{~dB}$ from $+3.0 \mathrm{~dB}$, leads to a drop of the maximum fidelity of nearly $32 \%$. 


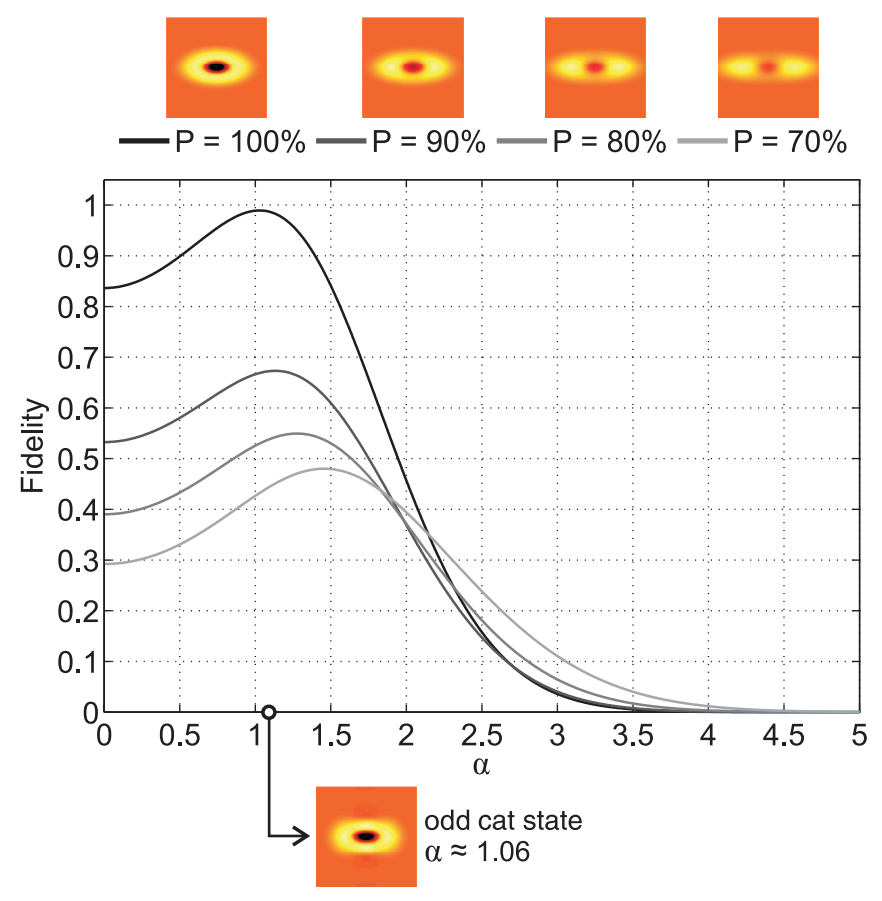

FIG. 3. (Color online) Fidelity between an ideal cat state $\left|\kappa_{-}(\alpha)\right\rangle$ and the small-amplitude cat states obtained fromthe squeezed vacuum $\xi_{p}=-3 \mathrm{~dB}$ at four squeezing purities $\mathcal{P}$ (marked in decreasing darkness of gray): $100 \%, 90 \%, 80 \%$, and $70 \%$. The Wigner functions of the PSSVs corresponding to these four purities are shown at the top. As a reference for what the "ideal" output ought to look like, the Wigner function of the state $\left|\kappa_{-}(1.06)\right\rangle$, which has the highest fidelity with the PSSV obtained from pure squeezing, is shown at the bottom. The transmission of the subtraction beam splitter is set at $99 \%$.

\section{AMPLIFICATION OF IDEAL ODD CAT STATES}

In Fig. 4 we present our amplification setup: Two identical cat states are mixed on a balanced beam splitter, whereupon one of the ensuing modes heralds the amplified output based on the measurement of $x$ quadratures around $x=0$. The scenario where the inputs are idealized cat states of opposite parity has been outlined by Takeoka and Sasaki in [31]. We will however look at the more practical case where the inputs have identical parity.

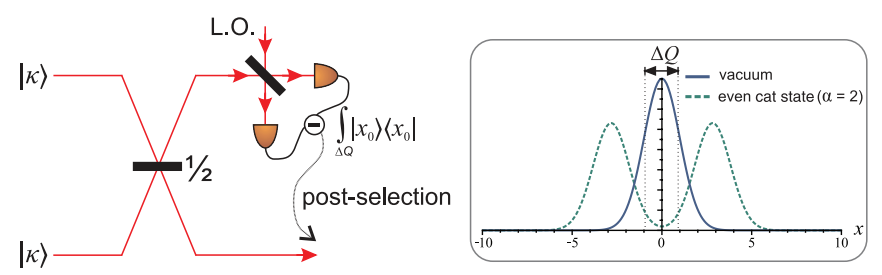

FIG. 4. (Color online) Setup for the amplification of two ideal Schrödinger cat states into a larger even cat state. The two inputs are mixed on a symmetric beam splitter and one of the outputs is projected onto an $x$ quadrature window of width $\Delta Q$ around $x_{0}=0$ by an otherwise ideal homodyne detector. Inset: Wave functions $\langle x \mid 0\rangle$ (solid curve) and $\left\langle x \mid \kappa_{+}(2)\right\rangle$ (dashed curve) of the vacuum and of an even cat state of size $\alpha=2$, respectively. The two functions are best distinguished at $x=0$ where their overlap is minimized.
Let us briefly run through the evolution of the state in this setup. We can readily see that the state emerging from the first balanced beam splitter contains an even cat state of amplitude $\sqrt{2}$ times larger:

$$
\left|\kappa_{ \pm}(\alpha)\right\rangle \otimes\left|\kappa_{ \pm}(\alpha)\right\rangle \rightarrow\left|\kappa_{+}(\sqrt{2} \alpha)\right\rangle \otimes|0\rangle \pm|0\rangle \otimes\left|\kappa_{+}(\sqrt{2} \alpha)\right\rangle .
$$

If, by measuring one of the modes, we can postselect $|0\rangle$ from $\left|\kappa_{+}(\sqrt{2} \alpha)\right\rangle$, then the other mode will collapse onto the desired amplified state $\left|\kappa_{+}(\sqrt{2} \alpha)\right\rangle$. The accuracy of this discrimination is of course limited by the overlap of the vacuum with the cat state $\left\langle 0 \mid \kappa_{+}(\sqrt{2} \alpha)\right\rangle$, which is however negligible for $\alpha \gg 0$. Based on the wave function profiles of the vacuum and the cat state, we can see that homodyne measurement of the $x=0$ quadrature is indeed a good way to discriminate the two states as it is where their overlap is minimized (see the inset of Fig. 4). The ambiguity of the discrimination is quantified in $\S \mathrm{A} 2$.

Applying the homodyne projection $\left|x_{2}=0\right\rangle\left\langle x_{2}=0\right|$ on the second mode of (4), we are left with

$$
\left|\psi_{\text {out }}\right\rangle=\frac{e^{\alpha^{2}}}{2} \frac{|\sqrt{2} \alpha\rangle+|-\sqrt{2} \alpha\rangle \pm 2 e^{-2 \alpha^{2}}|0\rangle}{\sqrt{\cosh \left(2 \alpha^{2}\right)+e^{-2 \alpha^{2}} \pm 2 e^{-\alpha^{2}}}} .
$$

The fidelity of this state with an ideal even cat state of size $\beta$ is

$$
F=\operatorname{sech}\left(\beta^{2}\right) \frac{\left|\cosh (\sqrt{2} \alpha \beta) \pm e^{-\alpha^{2}}\right|^{2}}{\cosh \left(2 \alpha^{2}\right)+e^{-2 \alpha^{2}} \pm 2 e^{-\alpha^{2}}} .
$$

In practice, a valid output is heralded whenever the homodyne detector records a state whose $x$ quadrature lies within a window $\Delta Q$ around $x=0$ where $\Delta Q$ will be expressed in shot-noise units (SNUs). ${ }^{3}$ We keep track of such a postselection window because no practical homodyning device has enough resolution to truly project onto an exact quadrature $\left|q_{0}\right\rangle\left\langle q_{0}\right|$. Experimentally, such a precise projection would not be desirable either for it would lead to very small success probabilities. A compromise is therefore to allow a finite postselection range. A full model of this realistic "wide" homodyning is presented in Appendix A 4.

From now on, we shall consider only odd cat inputs to the amplification setup. (The next section deals with approximations to odd-cat-state inputs, namely, PSSVs.) A contour plot of the fidelity between an even cat state of size $\beta$ and the amplified state from two odd cat states of size $\alpha$ is shown in Fig. 5 for a homodyning window of 1 SNU. The $\sqrt{2}$ amplification factor that was also witnessed in earlier schemes $[30,31,35]$ is recognizable as the slope $\frac{\beta}{\alpha}$ where the fidelity is optimized. The bend of this optimum crest for $\alpha \leqslant 1$ arises from the vacuum component which "survives" the postselection but vanishes from the output state $\left|\psi_{\text {out }}\right\rangle$ for larger $\alpha$. This feature is in a sense a manifestation of the discreteness of photon numbers for weak coherent states. As can be seen from (5), the output consists of an even cat state minus a vacuum. For $\alpha \rightarrow 0$, this "subtraction"

\footnotetext{
${ }^{3}$ By shot-noise unit, we mean the standard deviation of shot noise in phase space. By setting $\hbar=1$, this implies $1 \mathrm{SNU}=1 / \sqrt{2}$ (in absolute units of phase-space quadrature).
} 


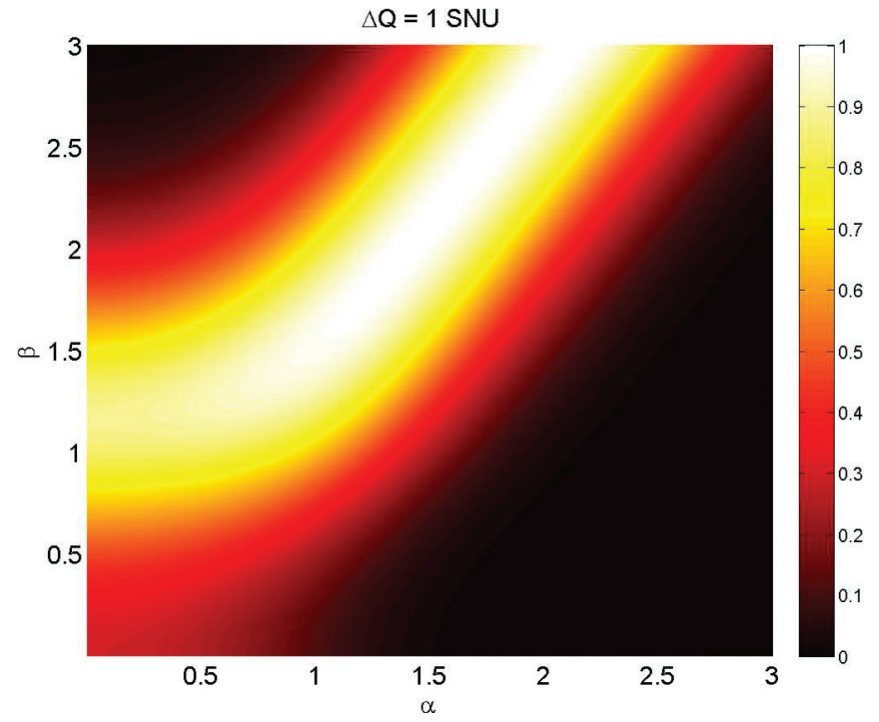

FIG. 5. (Color online) Contour plot of the fidelity between an even cat of size $\beta$ and the amplification obtained from two odd cats of size $\alpha$. The post-selection window is set to $1 \mathrm{SNU}$.

of the vacuum component yields a state whose two-photon component has a relatively higher weight than in any even cat state of size $\beta \ll 1$. The proportionality in amplitude between input and amplified cat states thus breaks down, and it is instead $|\beta \approx 1\rangle$ - of all even cat states - that exhibits the best fidelity with the output.

Just as in Fig. 2 for the PSSV state, it is informative to look at the mapping between input and output parameters which optimizes fidelity. This is shown in Fig. 6 where the optimal input-state amplitude is plotted as a function of the amplitude $\beta$ of the target state $\left|\kappa_{+}(\beta)\right\rangle$. For example, if one wants to produce an even cat state of size $\beta=2$, then an input odd cat
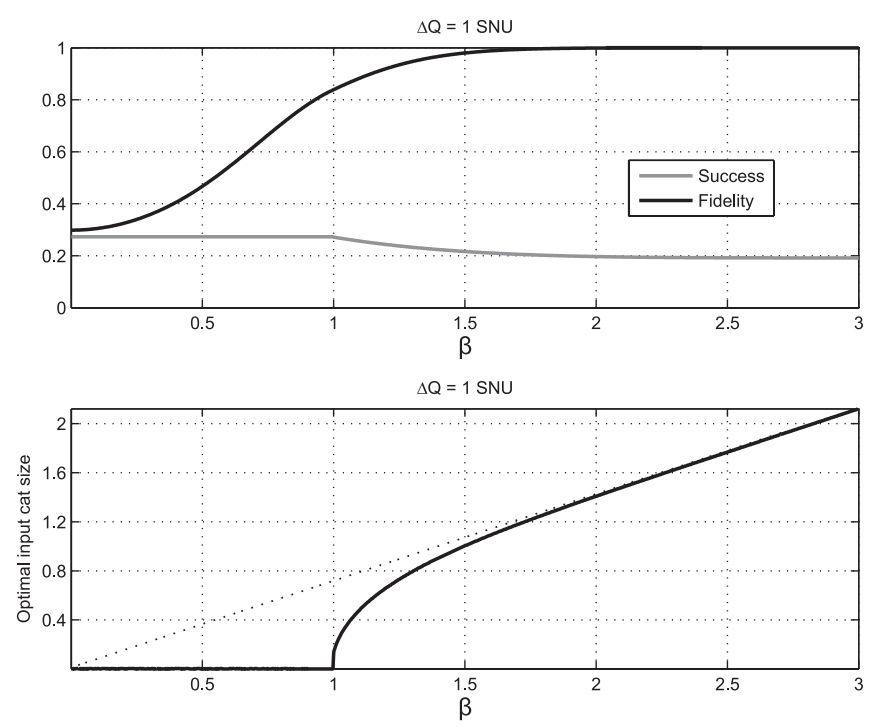

FIG. 6. Top: Maximum fidelity (black) and corresponding success probability (gray) of the amplified output with respect to an even cat state of size $\beta$. Bottom: Amplitude $\alpha$ of the input required to obtain the maximum fidelity of the output with an even cat state of size $\beta$. The dotted line marks the $\sqrt{2}$ amplification factor. The homodyne postselection window is $1 \mathrm{SNU}$ wide.

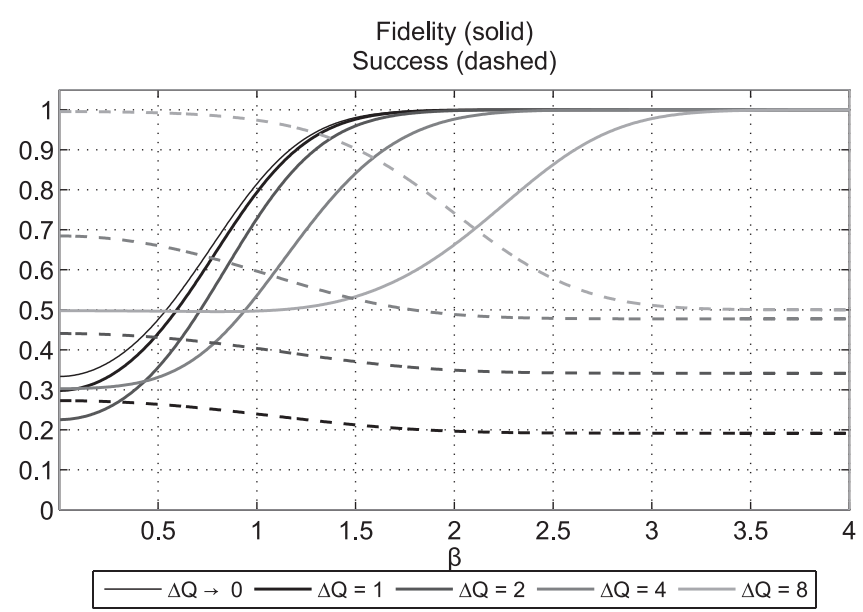

FIG. 7. Solid curves: Fidelity between an even cat state of size $\beta=\sqrt{2} \alpha$ and the output of mixing two odd cat states of size $\alpha$ for five homodyning widths $\Delta Q$ (in SNUs). Dashed curves: Corresponding success probabilities. (The curve for $\Delta Q \rightarrow 0$ is zero throughout since the probability of picking out the exact $x=0$ quadrature is vanishingly small.) The shading of the curves is lightened with larger homodyning windows.

state of size $\alpha=1.4$ is required. The fidelity of the output with $\left|\kappa_{+}(\beta=2)\right\rangle$ will be nearly $100 \%$ and the success probability of the operation about $20 \%$. As discussed above, the flat plateau for $\beta \leqslant 1$ corresponds to the range where the vacuum component that filtered through the postselection becomes predominant. The consequence is that the single-photon "cat state" $\left|\kappa_{-}(\alpha \rightarrow 0)\right\rangle \approx|1\rangle$ becomes the only input to optimize outputs of target size $\beta \in[0,1]$.

To assess the robustness of the amplification scheme to the homodyning width $\Delta Q$, Fig. 7 plots the fidelity between the output produced from two odd cat states $\left|\kappa_{-}(\alpha)\right\rangle$ and an even cat state $\left|\kappa_{+}(\beta=\sqrt{2} \alpha)\right\rangle$ given different homodyning windows. The fidelity curve for exact homodyning $\Delta Q \rightarrow 0$ is also plotted as a reference. It can be seen that the amplification is vulnerable only to $\Delta Q$ for small inputs. Beyond a target size of $\beta \approx 3.5$, homodyning widths of up to 8 SNUs hardly have any effect on the fidelity. From an experimental point of view, this robustness of the homodyning postselection allows one to reach higher success probabilities without compromising fidelity. For example, by sending in two odd cat states of size $\alpha=2.5$, one of every two homodyne measurements will successfully herald an even cat of size $\beta=\sqrt{2} \times 2.5 \approx 3.5$ with a fidelity of nearly $100 \%$.

For another perspective on the dependence of fidelity on homodyning width, let us consider the amplification of two odd cat states of a fixed size $\alpha=1$. The decrease in fidelity of the output with an even cat state of size $\beta=\sqrt{2}$ is traced in Fig. 8. Also shown are three sample Wigner profiles of the output, which display a clear degradation of the negativity as $\Delta Q$ increases.

The simulations presented above, as well as all other numerical results in this article are arrived at by a generic method of simulating linear transformation and projective measurements of states consisting of Gaussian superpositions (see Appendix A 3). 


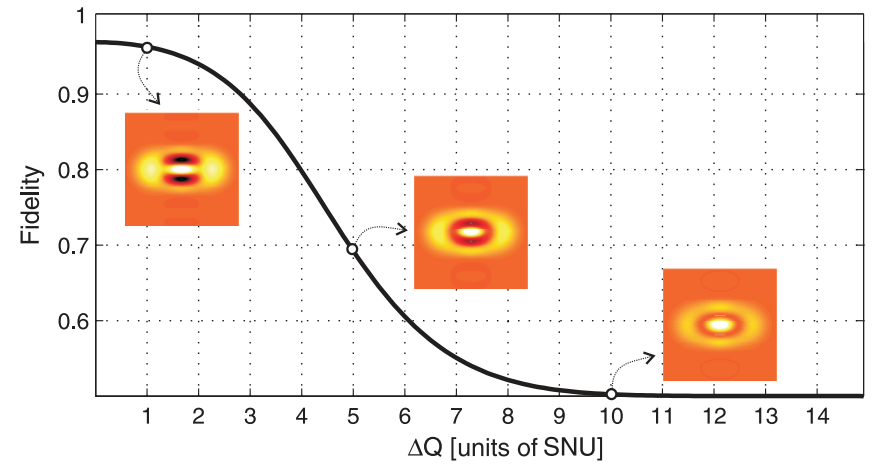

FIG. 8. (Color online) Fidelity of the state amplified from two cat states of size $\alpha=1$ with an even cat of size $\beta=\sqrt{2}$ for a homodyning window $\Delta Q \in[0,15]$ SNUs. The decrease in fidelity for wider homodyning windows is understandable from the increased overlap between the vacuum and the cat state, as illustrated in the inset of Fig. 4. The Wigner profile of the output is shown for three sample values of $\Delta Q$ at 1,5 , and 10 SNUs, respectively.

\section{AMPLIFICATION OF APPROXIMATE SMALL CAT STATES}

In this section, we consider the more realistic case where PSSVs are amplified, i.e., the inputs to Fig. 4 are the outputs of Fig. 1. In Fig. 9, the fidelity profile with an even cat state of size $\beta$ is plotted with respect to the input (pure) squeezing for $\Delta Q=1 \mathrm{SNU}$ and $T=95 \%$. The contour lines of PSSV generation are overlaid to visualize the amplification, i.e., the

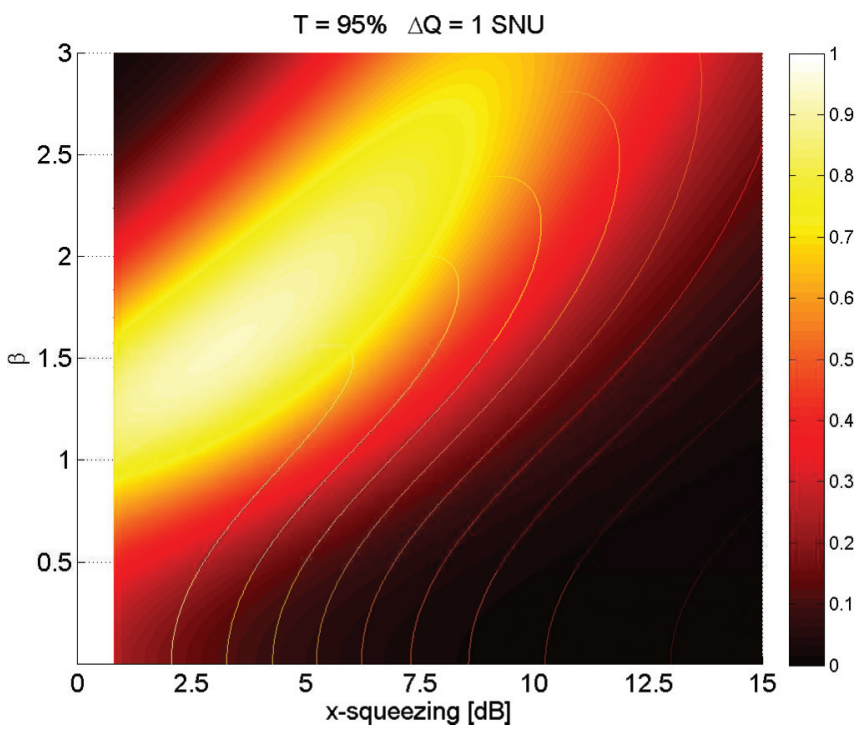

FIG. 9. (Color online) Contour plot of the fidelity between an even cat state $\left|\kappa_{-}(\beta)\right\rangle$ and the amplified PSSV obtained from an antisqueezing $\xi_{x}$ between 0 and $15 \mathrm{~dB}$. The transmission of the subtraction beam splitter is $95 \%$. The contour lines of PSSV generation fidelity are overlaid to better visualize the shift in target amplitude $\beta$ resulting from the amplification. The blank vertical stripe for $\xi_{x} \leqslant 0.8 \mathrm{~dB}$ is a region where numeric underflow is too frequent to produce reliable data. (This is because the normalization factor which enters in the fidelity is itself inversely proportional to the success probability. The latter tends to negligible values for small squeezing.)
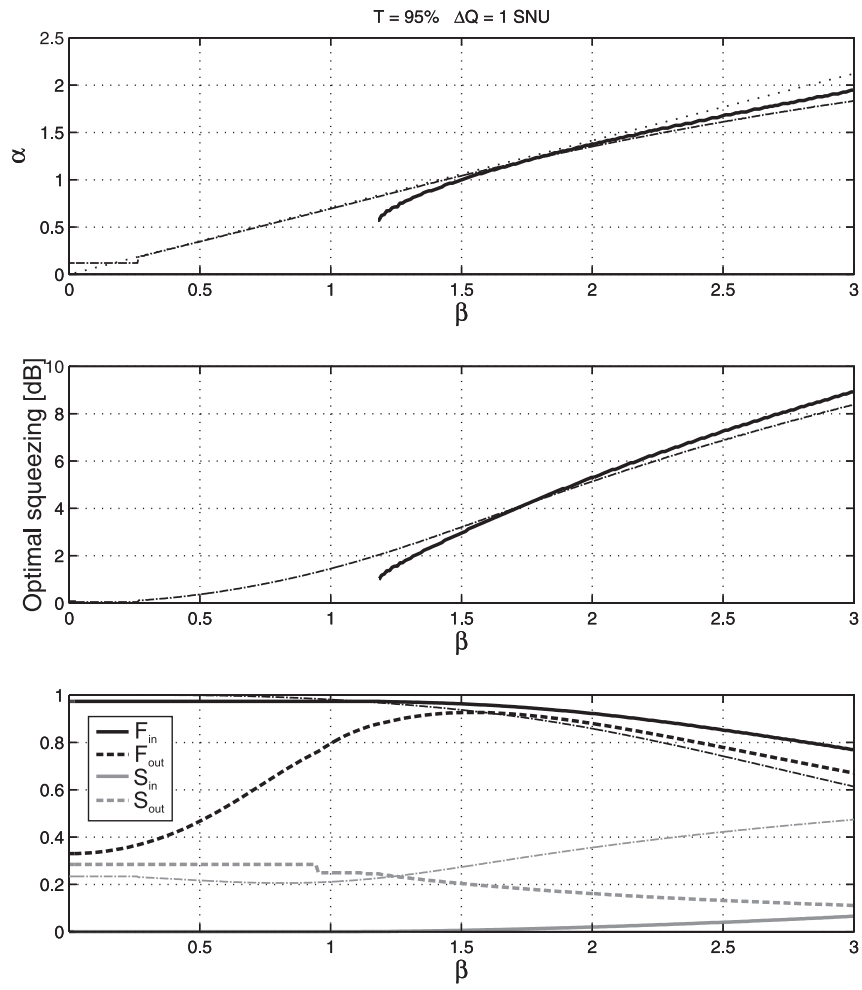

FIG. 10. Top: Dependence of the effective size $\alpha$ of the PSSVstate input on the target size $\beta$ of the output such that the fidelity of the output with $\left|\kappa_{+}(\beta)\right\rangle$ is maximized. The dotted line marks the $\alpha / \beta=1 / \sqrt{2}$ amplification ratio. The subtraction beam splitter for the PSSV generation is set to $95 \%$ and the homodyning window to 1 SNU. Middle: Pure squeezing required of the input in order to achieve the maximal fidelity with an even cat state of size $\beta$. Bottom: Maximum fidelity obtainable at the output with an even cat state of size $\beta$ (dashed black), along with the corresponding success probability of the amplification (dashed gray). Also shown is the maximum fidelity of the required input PSSV with an odd cat state of size $\alpha$ (solid black), and the success probability of the PSSV generation (solid gray). (In all three graphs, the finer dash-dotted lines refer to the results of Lund et al. in [30], with, in the bottom graph, the black and gray shadings representing fidelity and success probability, respectively.)

shift of the high-fidelity area upwards to larger values of $\beta-\mathrm{cf}$. Fig. 2.

In order to assess the performance of the amplification scheme, we shall set a target even cat state of amplitude $\beta$ and retrace the quantum circuit to see what input squeezing is necessary to achieve the highest fidelity with $\left|\kappa_{+}(\beta)\right\rangle$ at the output. This is shown in Fig. 10, along with the dependence of the success probability and fidelity on $\beta$, as well as the size $\alpha$ of the odd cat state that best matches the input PSSV. Let us assume, for instance, that we want to produce an even cat state of size $\beta=1.5$. The required squeezing for the PSSV will then be around $2.9 \mathrm{~dB}$, corresponding to a fidelity of $96.4 \%$ with an odd cat state of size $\alpha \approx 1.0$. The output, however, will have a fidelity of $92.6 \%$ with $\left|\kappa_{+}(\beta=1.5)\right\rangle$ and the success probability of the amplification will be $20 \%$ (notwithstanding the success probability of $0.6 \%$ required to produce the "offline" PSSV).

Note that the solid curves in the first two plots of Fig. 10 only start at $\beta \approx 1.2$. This is because below that threshold, 
the optimal squeezing lies in a numerically unstable region marked the blank band in Fig. 9.

A first-hand observation to be drawn from Fig. 10 is the similarity it shows with Fig. 6 for small target cat-state sizes. Both scenarios with ideal cat states and PSSV inputs start out with a fidelity around $30 \%-35 \%$ which then increases to over $90 \%$ for target cat states of size $\beta \approx 1.5$. Beyond this point, however, the performance of PSSV amplification starts to degrade, whereas that of ideal cat states can be pursued to indefinitely large target sizes while retaining unit fidelity. One conclusion is therefore that the approximation of cat states from PSSVs can be used to generate amplified states of sizes up to $\beta=1.5$ with practically the same fidelity as if one used ideal odd cat states as inputs. On the other hand, both Figs. 6 and 10 exhibit the plateau in optimal input cat-state size-or in the case of PSSVs, effective cat-state size-which we discussed in the previous section. In that region, $\beta \in[0,1]$, the ideal input cat states or PSSVs cease to have any dependence on the target size $\beta$ and discrete states, namely, single photons, become the optimal input state.

In addition to simulating our own amplification scheme, we have overlaid as finer dash-dotted curves the results of Lund, Jeong, Ralph, and Kim, which we shall refer to as the LJRK scheme [30]. Instead of using homodyning, they mix the heralding arm with a coherent state on a balanced beam splitter such that both emerging modes contain at least one photon if the heralded mode is amplified. In contrast to the homodyne method, the LJRK projection is unambiguous as it does not yield any residual vacuum component, unlike in (5). This explains why the fidelity in the LJRK scheme remains quasi-ideal for low target sizes $\beta$. Beyond $\beta \approx 1.5$,
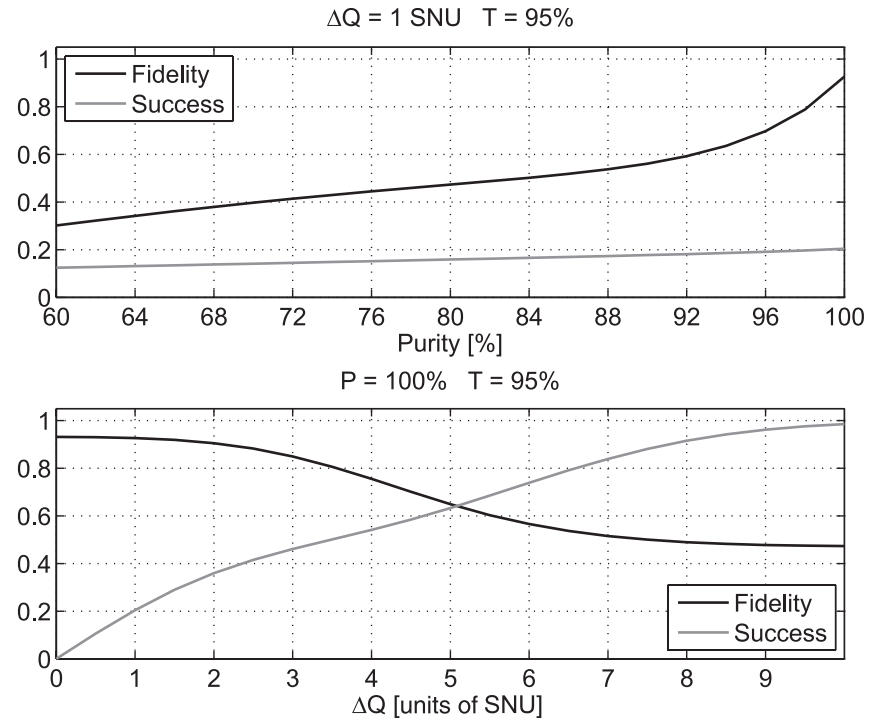

FIG. 11. Robustness of the PSSV amplification to squeezing impurity (top) and homodyning width (bottom). The fidelity with an even cat state of size $\beta=1.5$ and the success probability of the amplification (assuming offline PSSVs) are plotted in black and gray, respectively. The transmission of the subtraction beam splitter from which the small-amplitude cat states are generated is $95 \%$ and the antisqueezing in $p$ is fixed, $\xi_{p}=-2.9$. [This squeezing is chosen such that the fidelity with $\left|\kappa_{+}(\beta=1.5)\right\rangle$ is maximized in the case of pure squeezing and $\Delta Q=1 \mathrm{SNU}$-cf. the middle plot of Fig. 10.] however, both methods are comparable in terms of fidelity and amplification factor. The homodyne method offers nonetheless experimental advantages over the photon detection of LJRK in that it does not suffer as much of quantum inefficiency or electronic noise. In particular, the need for coincident detection of photons in the LJRK scheme suppresses the success probability by the square of the quantum inefficiency. (The dash-dotted curve in Fig. 10 assumes ideal quantum efficiency.)

If one assumes ideal quantum efficiencies at the detectors, the two main nuisances in the performance of the amplification scheme are (i) the impurity $\mathcal{P}$ of squeezing and (ii) the width $\Delta Q$ of the homodyning detection. To visualize the robustness of the scheme to these two factors, let us choose an optimal input squeezing with a target even cat state of size, say, $\beta=$ 1.5. From Fig. 10, this corresponds to $\xi_{p}=-2.9 \mathrm{~dB}$ for a fidelity of $92.6 \%$ and a success probability (assuming offline PSSVs) of $20 \%$. From this reference point, the trend of fidelity and success probability on varying either $\mathcal{P}$ or $\Delta Q$ is plotted in Fig. 11. Recall that, by convention, we model a decrease of purity by an increase in antisqueezing $\left(\xi_{x}\right)$ while maintaining squeezing proper $\left(\xi_{p}\right)$ fixed.

\section{SCALABILITY}

As was shown in the previous section, we are bound by a trade-off between amplification and fidelity. That is, if the output fidelity is to be kept high, one has to work with smaller small-amplitude cat states to begin with, and thus cannot reach higher amplitudes (cf. Fig. 10). A high fidelity can, however, be achieved with larger states if we cascade the setups into a complete binary tree. We shall discuss the scalability of such a recursive amplification in the case of perfect homodyning $(\Delta Q \rightarrow 0)$ and pure squeezing $(\epsilon=1)$, both for ideal input cat states and for PSSV inputs.

Let us describe each iteration stage $k$ as the amplification of two identical wave functions $\varphi_{k}\left(x_{1}\right)$ and $\varphi_{k}\left(x_{2}\right)$ into a larger state $\varphi_{k+1}\left(x_{1}\right)$ where mode 2 has been postselected by homodyning. This iteration step is given by

$$
\begin{aligned}
\varphi_{k}\left(x_{1}\right) \varphi_{k}\left(x_{2}\right) & \rightarrow\{50: 50 \text { beam splitting }\} \\
& =\varphi_{k}\left(\frac{x_{1}-x_{2}}{\sqrt{2}}\right) \varphi_{k}\left(\frac{x_{1}+x_{2}}{\sqrt{2}}\right) \\
& \rightarrow\left\{\text { projection on }\left|x_{2}=0\right\rangle\left\langle x_{2}=0\right|\right\} \\
& =\varphi_{k}\left(\frac{x_{1}}{\sqrt{2}}\right) \varphi_{k}\left(\frac{x_{1}}{\sqrt{2}}\right), \\
\varphi_{k+1}\left(x_{1}\right) & \propto \varphi_{k}^{2}\left(\frac{x_{1}}{\sqrt{2}}\right),
\end{aligned}
$$

where the initial wave function $\varphi_{0}$ is that of the inputs. It can be obtained from the one-variable analog of (A16). In the case of input PSSVs, this involves the highly unbalanced mixing of two vacua, one of which is squeezed, and-ideally-a onephoton projector $\varphi_{|1\rangle}$. That is,

$$
\begin{aligned}
\varphi_{0}\left(x_{1}\right)= & \lim _{T \rightarrow 1} \int_{-\infty}^{\infty} \varphi_{\hat{S}|0\rangle}\left(\sqrt{T} x_{1}-\sqrt{1-T} x_{2}\right) \\
& \times \varphi_{|0\rangle}\left(\sqrt{1-T} x_{1}+\sqrt{T} x_{2}\right) \varphi_{|1\rangle}^{*}\left(x_{2}\right) d x_{2} .
\end{aligned}
$$




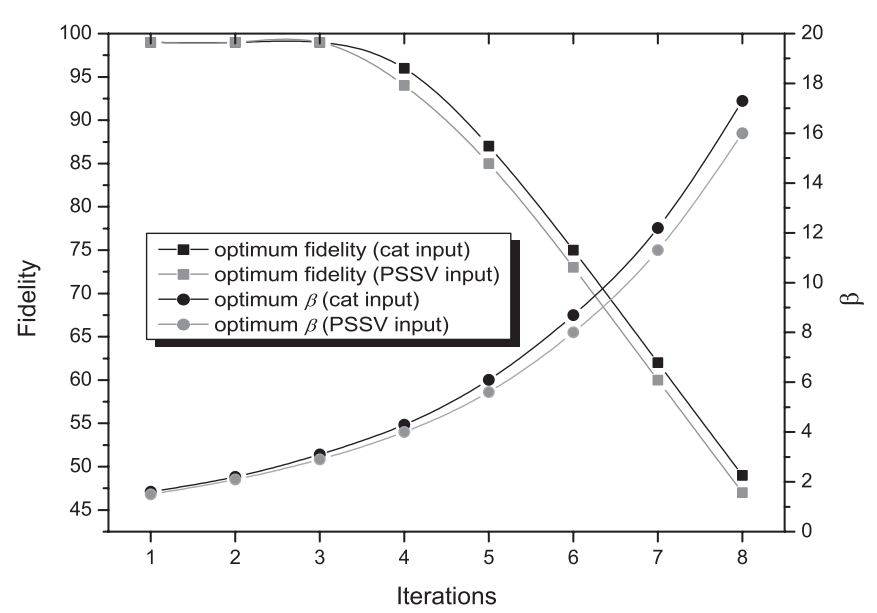

FIG. 12. Maximum fidelity (squares) and corresponding effective size $\beta$ (disks) of the output as a function of the number of iterations. The inputs to the first iteration are an ideal odd cat state of size $\alpha=1$ (black) or a PSSV squeezed by $3 \mathrm{~dB}$ (gray).

Since we only want to investigate how scaling behaves, however, we shall simplify the PSSV by a squeezed single photon,

$$
\lim _{T \rightarrow 1} \varphi_{0}(x)=\langle x|\hat{S}| 1\rangle=\frac{\sqrt{2}}{\pi^{1 / 4} s^{3 / 2}} x e^{-x^{2} / 2 s^{2}},
$$

where $s=10^{\xi / 20}$ is the factor by which the quadrature phase space is rescaled as a consequence of the pure squeezing (i.e., $x \rightarrow s x$ and $p \rightarrow p / s)$.

The wave function of the state at the $k$ th iteration is given in closed form by

$$
\varphi_{k}(x)=\frac{1}{\mathcal{N}^{(k)}} \varphi_{0}^{2 k}\left(2^{-k / 2} x\right),
$$

where

$$
\mathcal{N}^{(k)}=\left[\int_{-\infty}^{\infty} \varphi_{0}^{2 k}\left(2^{-k / 2} x\right) d x\right]^{1 / 2}
$$

is the normalization factor.

Figure 12 tracks both the amplitude and the fidelity of the outputs as a function of the number of iterations $k$. As already expected from (10), the output amplitude grows as $\sqrt{2}^{k}$. This does not imply, however, that iterations could be carried out indefinitely. For the particular choice of initial conditions plotted, $\alpha=1$ for the cat state or $3 \mathrm{~dB}$ of squeezing for the PSSV, the fidelity drops below $90 \%$ at the fifth iteration. This shortcoming of recursive amplifications is due to the nonunitarity of the amplification in the coherent-state basis. Looking back at the idealized case of (5), we see that the amplification is not a straightforward mapping of a cat state onto a larger one, but instead introduces an extra vacuum term. This vacuum, which arises from the intrinsic ambiguity of the homodyne projection, is also amplified along with the cat state. Even if one does start the first iteration with an ideal cat state, any subsequent iteration $k$ will inherit this vacuum component, which in turn will contaminate the following step $k+1$ with additional terms orthogonal to an even cat state.

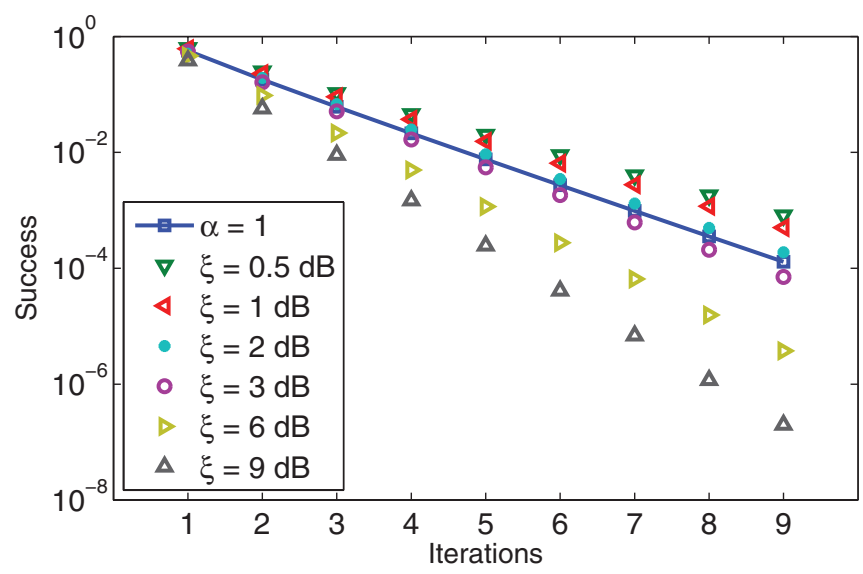

FIG. 13. (Color online) Success probability of iterated amplifications for an input cat state $\left|\kappa_{-}(\alpha=1)\right\rangle$ as well as for various input PSSVs with squeezing $\xi \in\{0.5,1,2,3,6,9\} \mathrm{dB}$.

The success rate of iterated amplifications is the main issue facing scalability. As shown in Fig. 13, the scheme exhibits a sharp drop in the success probability with the increasing number $k$ of iterations. Another obvious overhead is the number of input states which grows as $2^{k}$.

\section{CONCLUSION}

We have presented an amplification protocol for cat states that is based on imprecise homodyne measurement. The performance of the scheme was assessed in terms of fidelity and success rate and illustrated by an optimized relation between the target size $\beta$ and the input size $\alpha$ of the cat states involved. Given that ideal cat states are challenging to produce, we also presented how the amplification behaves with approximations to cat states, namely, photon-subtracted squeezed vacuum. Here again, we determined the optimal relation between the input squeezing and the effective size of the output. The purity of squeezing at the input was determined to be crucial in achieving a high fidelity at the output. The amplification was, however, relatively robust to imprecise homodyne thresholding, thereby allowing an increase in success probability.

The recursive application of the amplification protocol is then simulated in the idealized case of exact homodyning and pure squeezing. Although the amplification factor does grow as $\sqrt{2}^{k}$ with the number $k$ of recursions, the output fidelity eventually degrades due to the nonunitarity of the amplification. One is therefore constrained to a finite number of recursions where the state is amplified while at the same time retaining a high fidelity.

We saw in Sec. IV that target cat-state sizes $\beta=1.5$ can be reached with fidelities up to $93 \%$ if one uses PSSVs obtained from a 5\% tapped-off squeezed vacuum. (If one assumes ideally squeezed single photons, that fidelity can even increase to $98 \%$; cf. the first iteration in Fig. 12. For higher iterations of the amplification, amplitudes of $\beta \approx 3$ can be obtained while maintaining fidelity around 98\%.) Although such approximate states may not permit fault-tolerant quantum computation, they nonetheless allow for proof-of-principle 
experiments that require effective cat-state sizes larger than those of basic PSSVs. For instance, our amplification protocol can find uses in teleportation [36] or some demonstrations of quantum gates [5,37]. The question of whether the amplified states can be harnessed for any particular use in quantum information will require a feasibility study of its own that pays particular attention to the trade-off between target sizes and fidelities.

Let us conclude with a final note on the practical challenges to our protocol. In addition to the issues proper to PSSV preparation (and already discussed in [19-21,24,25]), the key challenge facing the amplification protocol is phase stability. This arises because the pairs of interfering PSSVs need to be synchronous, thereby leading to very small success rates. This is to be factored on top of the already small success rate of an otherwise ideal postselection (Fig. 13). Small success rates are compensated for by running the experiment over a larger batch of input states. However, this in turns requires that the relative phases - of the two interfering PSSVs and of the local oscillator-be kept stable for protracted periods using a particularly reliable locking system.

\section{APPENDIX: MATHEMATICAL DERIVATIONS}

\section{Expression for the purity of the squeezed vacuum}

Let the variance of the vacuum phase-space distribution be labeled by $V_{0}$. As will be discussed more formally in Appendix A 3, the squeezing operation along a quadrature $q$ consists of a rescaling of phase space $q \rightarrow s_{q} q$ where $s_{q}=\sqrt{V_{q} / V_{0}}$ such that the new variance along $q$ is $V_{q}$. The relationship between the dimensionless rescaling factor $s_{q}$ and the squeezing $\xi_{q}$ (in decibels) is given by

$$
\xi_{q}=10 \log _{10} \frac{V_{q}}{V_{0}}=20 \log _{10} s_{q} \Leftrightarrow s_{q}=10^{\xi_{q} / 20} .
$$

The purity of a state of Wigner function $W$ is given by

$$
\mathcal{P}=2 \pi \iint W^{2} d x d p,
$$

and the Wigner function of a squeezed vacuum state $\hat{S}|0\rangle$ is

$$
W_{\hat{S}|0\rangle}(x, p)=\frac{1}{\pi s_{x} s_{p}} e^{-\left(x / s_{x}\right)^{2}-\left(p / s_{p}\right)^{2}} .
$$

The purity of the squeezed vacuum can therefore be shown to be

$$
\mathcal{P}=\frac{1}{s_{x} s_{p}}=10^{-(1 / 20)\left(\xi_{x}+\xi_{p}\right)} .
$$

\section{Error in the discrimination between the vacuum and a cat state with a homodyne projector}

As mentioned in Sec. III, the purpose of the homodyne measurement is to collapse the output state onto an even

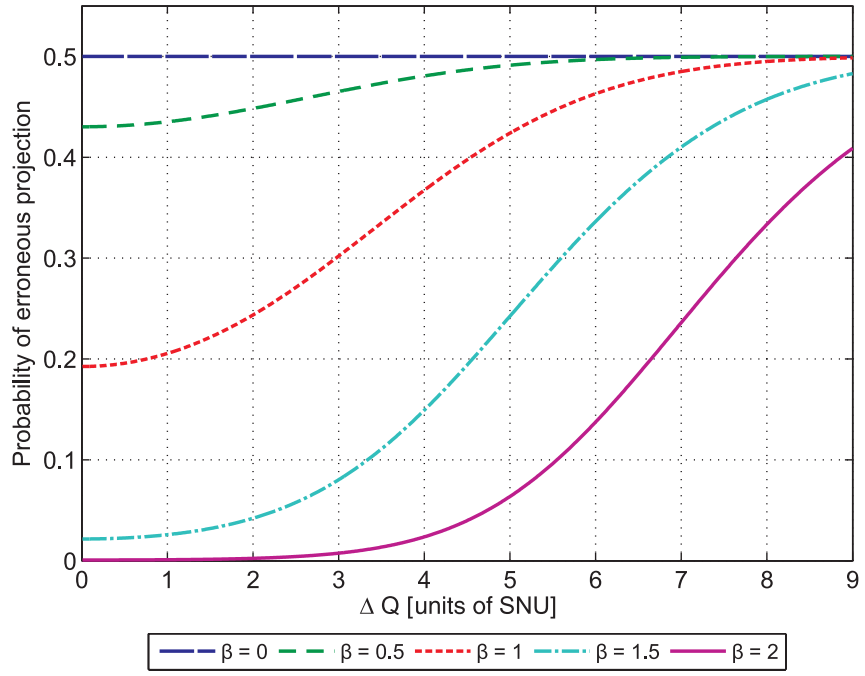

FIG. 14. (Color online) Probability $P_{\text {err }}$ of mistaking an even cat state of size $\beta$ for a vacuum state as a function of the quadrature acceptance window $\Delta Q$.

cat state upon the detection of vacuum; cf. Eq. (4) and the inset of Fig. 4. The projector for this measurement is

$$
\hat{\Pi}_{|0\rangle}^{\mathrm{HD}}=\int_{-\frac{1}{2} \Delta Q}^{\frac{1}{2} \Delta Q}|x\rangle\langle x| d x .
$$

Due to the intrinsic overlap of the wave functions of $|0\rangle$ and $\left|\kappa_{+}(\alpha)\right\rangle, \hat{\Pi}_{|0\rangle}^{\mathrm{HD}}$ can act only as an approximate discriminator between them. In addition, the finite width of the quadratureselection window introduces an approximation of its own. The error in the discrimination, namely, the probability of mistaking an even cat state for the vacuum is given by

$$
P_{\mathrm{err}}=\frac{P\left(\left|\kappa_{+}(\beta)\right\rangle\right)}{P\left(\left|\kappa_{+}(\beta)\right\rangle\right)+P(|0\rangle)},
$$

where $P(|\gamma\rangle)=\operatorname{Tr}\left\{\hat{\Pi}_{|0\rangle}^{\mathrm{HD}}|\gamma\rangle\langle\gamma|\right\}$ and $|\gamma\rangle \in\left\{|0\rangle,\left|\kappa_{+}(\beta)\right\rangle\right\}$. Figure 14 shows that the discrimination is best achieved for small $\Delta Q$ and large $\beta$. The asymptotic convergence to $P_{\text {err }}=0.5$ at large $\Delta Q$ or small $\beta$ indicates a complete lack of discrimination between the states: They become equally likely to be inferred by the homodyne projector.

\section{Linear transformations and measurements with Gaussian states and operations}

In this section, we shall present the calculational tools we have used to perform our simulations. All the quantum states and operations involved in this article are made up of Gaussian superpositions in the Wigner picture. That is, any state $\hat{\rho}$ or measurement operator $\hat{\Pi}$ can be written in the Wigner picture as a function

$$
W(x, p)=\sum_{i} G_{i}(x, p)
$$


where $G$ is a Gaussian function in both quadratures,

$G(x, p)=g_{0} \exp \left(g_{1} x^{2}+g_{2} x+g_{3} x p+g_{4} p^{2}+g_{5} p+g_{6}\right)$,

with constant coefficients $g_{k} \in \mathbb{C}: k \in\{0, \ldots, 6\}$ and $g_{1}, g_{4} \in$ $\mathbb{R}^{-}$. For example, a cat state is made up of four such Gaussians,

$$
W_{\left|\kappa_{ \pm}(\gamma)\right\rangle}(x, p)=\sum_{j=1}^{4} G_{\kappa_{ \pm}}^{(j)}(x, p, \gamma)
$$

where

$$
\begin{aligned}
& G_{\kappa_{ \pm}}^{(1)}(x, p)=-\frac{e^{-x^{2}-p^{2}+2 \sqrt{2} i \gamma p}}{2 \pi\left(-e^{-2 \gamma^{2}} \mp 1\right)}, \\
& G_{\kappa_{ \pm}}^{(2)}(x, p)=-\frac{e^{-x^{2}-p^{2}-2 \sqrt{2} i \gamma p}}{2 \pi\left(-e^{-2 \gamma^{2}} \mp 1\right)}, \\
& G_{\kappa_{ \pm}}^{(3)}(x, p)=\frac{e^{-x^{2}-p^{2}+2 \sqrt{2} \gamma x-2 \gamma^{2}}}{2 \pi\left(-e^{-2 \gamma^{2}} \mp 1\right)}, \\
& G_{\kappa_{ \pm}}^{(4)}(x, p)=\frac{e^{-x^{2}-p^{2}-2 \sqrt{2} \gamma x-2 \gamma^{2}}}{2 \pi\left(-e^{-2 \gamma^{2}} \mp 1\right)},
\end{aligned}
$$

whereas the APD operation $\hat{\Pi}^{(\mathrm{APD})}=\hat{\mathbb{I}}-|0\rangle\langle 0|$ is made up of only two Gaussians,

$$
W_{\hat{\Pi}^{(\mathrm{APD})}}(x, p)=\sum_{j=1}^{2} G_{\hat{\Pi}^{(\mathrm{APD}, j)}}(x, p),
$$

where

$$
\begin{aligned}
G_{\hat{\Pi}^{(\mathrm{APD}, 1)}}(x, p) & =\frac{1}{2 \pi}, \\
G_{\hat{\Pi}^{(\mathrm{APD}, 2)}}(x, p) & =-\frac{1}{\pi} e^{-x^{2}-p^{2}} .
\end{aligned}
$$

If we now assume that all transformations undergone by the state $\hat{\rho}$ prior to measurement are linear, as is the case in the setups presented in this article, then the generic Gaussian form (A8) is conserved and only the quadrature vector

$$
\vec{q}=\left(x_{1}, p_{1}, \ldots, x_{N}, p_{N}\right)^{T}
$$

of the $N$ modes involved is transformed by a linear mapping

$$
\vec{q} \rightarrow \mathbf{A} \cdot \vec{q}
$$

where the $2 N \times 2 N$ matrix $\mathbf{A}$ is determined by the linear assemblage of passive elements making up the circuit, e.g., beam splitters, phase shifters, squeezers, etc.

Consider the generic quantum circuit depicted in Fig. 15 where once again we have only Gaussian states and operators as defined by (A7) and (A8). The effect of a re-scaled

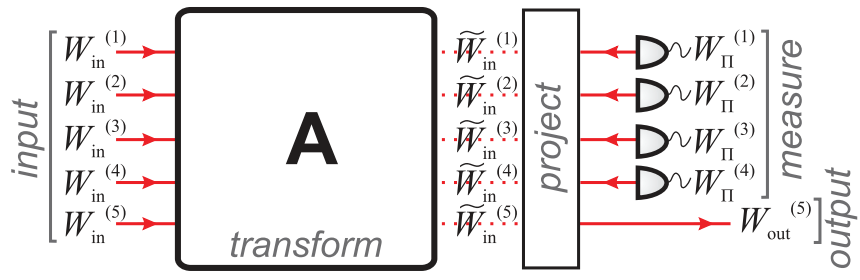

FIG. 15. (Color online) Sketch of a black box quantum circuit made up of $N=5$ input modes, $M=4$ measurement modes, and $N-M=1$ output modes. Each state occupying mode $k$ is represented by a Wigner function $W^{(k)}$. The input $\prod_{n}^{N} W_{\text {in }}^{(n)}$ is mapped according to (A15) into a linearly transformed state $\prod_{n}^{N} \tilde{W}_{\text {in }}^{(n)}$ which is then projected onto a "measurement state" $\prod_{m}^{M} W_{\hat{\Pi}}^{(m)}$.

quadrature space (A14) on a given Wigner function is equivalent to leaving the space unchanged while submitting the function to the inverse transformation. We shall label the transformed multimode Wigner function by a tilde such that the mapping (A14) yields

$$
W(\vec{q}) \rightarrow W\left(\mathbf{A}^{-1} \vec{q}\right)=\tilde{W}(\vec{q})
$$

If we now apply the measurement operators over $M<$ $N$ modes, the output over the remaining $N-M$ modes is given by

$$
W_{\hat{\rho}_{\text {out }}}=\iint_{\mathbb{R}} \prod_{n}^{N} \tilde{W}_{\hat{\rho}_{\text {in }}}^{(n)} \cdot \prod_{m}^{M} W_{\hat{\Pi}}^{(m)} d x_{m} d p_{m}
$$

where the superscript denotes the $k$ th mode.

Note that the number of output modes equals the number of input modes $N$ minus the number of measured modes $M$. If $M=N$, then there is no output state and the inner product (A16) leaves us with a scalar representing the success probability — or norm — of the projection of input states onto the measurement operators. Alternatively, this number could be interpreted as the fidelity between the overall states at either side of the diagram in Fig. 15.

The rationale for (A16) is valid regardless of whether the Wigner functions are Gaussian superpositions: From a mathematical standpoint, projective measurements are inner products between the measured state and the measuring operator. In functional analysis, this translates to an integral of the product of two states - the measured and the measuring stateover the entire phase space of measurement. The simplicity that comes from using (A16) with Gaussian superpositions is due to the fact that products and integrals of Gaussians are also still Gaussian.

\section{Wide homodyne measurement}

A homodyne measurement corresponds to a projection on a given quadrature $\left|q_{0}\right\rangle\left\langle q_{0}\right|$ where $\left|q_{0}\right\rangle=\cos \theta\left|x_{0}\right\rangle+\sin \theta\left|p_{0}\right\rangle$. This projector, when applied to a state whose Wigner function is $W(x, p)$, "picks out" the cross section along $q_{0}$ to yield a probability density. Such a projection is given in the quadrature 
basis by

$$
\hat{\Pi}_{\mathrm{HD}}\left(q_{0}\right)=\left|q_{0}\right\rangle\left\langle q_{0}\right|,
$$

which, in the Wigner representation, would most appropriately be given by the Dirac $\delta$ function

$$
W_{\mathrm{HD}}\left(q_{0}\right)=\delta\left(q-q_{0}\right) .
$$

If we choose for simplicity that $q_{0}=x_{0}$, then the projection operation (A16) onto some state $W(x, p)$ yields

$$
\begin{aligned}
& \iint_{\mathbb{R}} W(x, p) W_{\mathrm{HD}}\left(q_{0}\right) \mathrm{d} x \mathrm{~d} p \\
& \quad=\iint_{\mathbb{R}} W(x, p) \delta\left(x-x_{0}\right) \mathrm{d} x \mathrm{~d} p \\
& \quad=W\left(x_{0}, p\right)=P\left(x_{0}\right) .
\end{aligned}
$$

Intuitively, the probability of measuring exactly the quadrature $q_{0}$ should be vanishingly small. Yet $P\left(x_{0}\right)$ has a finite value since it is a probability density. To model the more realistic scenario where homodyne detection has a given resolution bandwidth $\Delta q$ around the measured value $q_{0}$, an interval projector is more appropriate:

$$
\hat{\Pi}_{\mathrm{HD}}\left(q_{0}, \Delta q\right)=\int_{q_{0}-\frac{\Delta q}{2}}^{q_{0}+\frac{\Delta q}{2}}|q\rangle\langle q| d q
$$

or, in Wigner representation,

$$
\begin{aligned}
& W_{\mathrm{HD}}\left(q_{0}, \Delta q\right) \\
& =\frac{1}{2 \pi} \int_{q_{0}-\frac{\Delta q}{2}}^{q_{0}+\frac{\Delta q}{2}} \delta\left(q-q^{\prime}\right) d q^{\prime} \\
& =\frac{1}{2 \pi}\left[\Theta\left(q-q_{0}+\frac{1}{2} \Delta q\right)-\Theta\left(q-q_{0}-\frac{1}{2} \Delta q\right)\right],
\end{aligned}
$$

where $\Theta$ is the Heaviside step function. Note how (A21) does not fit the Gaussian form of (A8). For calculational ease, the wide homodyning operation is therefore best performed last so as to maintain the convenient Gaussianity of the quantum states as long as possible.

In the limiting case of ideal homodyning, $\Delta Q \rightarrow 0$, we expect (A18) and (A21) to yield the same result.
[1] P. T. Cochrane, G. J. Milburn, and W. J. Munro, Phys. Rev. A 59, 2631 (1999).

[2] J. S. Neergaard-Nielsen, M. Takeuchi, K. Wakui, H. Takahashi, K. Hayasaka, M. Takeoka, and M. Sasaki, Phys. Rev. Lett. 105, 053602 (2010).

[3] T. C. Ralph, A. Gilchrist, G. J. Milburn, W. J. Munro, and S. Glancy, Phys. Rev. A 68, 042319 (2003).

[4] A. Gilchrist, K. Nemoto, W. J. Munro, T. C. Ralph, S. Glancy, S. L. Braunstein, and G. J. Milburn, J. Opt. B: Quantum Semiclass. Opt. 6, S828 (2004).

[5] A. Tipsmark, R. Dong, A. Laghaout, P. Marek, M. Jezek, and U. L. Andersen, Phys. Rev. A 84, 050301 (2011).

[6] T. C. Ralph, Phys. Rev. A 65, 042313 (2002).

[7] W. J. Munro, K. Nemoto, G. J. Milburn, and S. L. Braunstein, Phys. Rev. A 66, 023819 (2002).

[8] J. Joo, W. J. Munro, and T. P. Spiller, Phys. Rev. Lett. 107, 083601 (2011).

[9] H. Jeong, Phys. Rev. A 78, 042101 (2008).

[10] M. Stobińska, H. Jeong, and T. C. Ralph, Phys. Rev. A 75, 052105 (2007).

[11] C.-W. Lee and H. Jeong, Phys. Rev. A 80, 052105 (2009).

[12] G. McKeown, M. G. A. Paris, and M. Paternostro, Phys. Rev. A 83, 062105 (2011).

[13] B. Yurke and D. Stoler, Phys. Rev. Lett. 57, 13 (1986).

[14] A. Mecozzi and P. Tombesi, Phys. Rev. Lett. 58, 1055 (1987).

[15] H. Jeong, M. S. Kim, T. C. Ralph, and B. S. Ham, Phys. Rev. A 70, 061801 (2004).

[16] S. Glancy and H. M. de Vasconcelos, J. Opt. Soc. Am. B 25, 712 (2008).

[17] M. Dakna, T. Anhut, T. Opatrný, L. Knöll, and D.-G. Welsch, Phys. Rev. A 55, 3184 (1997).

[18] J. Wenger, R. Tualle-Brouri, and P. Grangier, Phys. Rev. Lett. 92, 153601 (2004).

[19] A. Ourjoumtsev, R. Tualle-Brouri, J. Laurat, and P. Grangier, Science 312, 83 (2006).
[20] T. Gerrits, S. Glancy, T. S. Clement, B. Calkins, A. E. Lita, A. J. Miller, A. L. Migdall, S. W. Nam, R. P. Mirin, and E. Knill, Phys. Rev. A 82, 031802 (2010).

[21] J. S. Neergaard-Nielsen, B. M. Nielsen, C. Hettich, K. Mølmer, and E. S. Polzik, Phys. Rev. Lett. 97, 083604 (2006).

[22] H. Jeong, A. P. Lund, and T. C. Ralph, Phys. Rev. A 72, 013801 (2005).

[23] A. P. Lund, T. C. Ralph, and H. L. Haselgrove, Phys. Rev. Lett. 100, 030503 (2008).

[24] K. Wakui, H. Takahashi, A. Furusawa, and M. Sasaki, Opt. Express 15, 3568 (2007).

[25] A. Ourjoumtsev, H. Jeong, R. Tualle-Brouri, and P. Grangier, Nature (London) 448, 784 (2007).

[26] A. E. B. Nielsen, and Klaus Mølmer, Phys. Rev. A 76, 043840 (2007).

[27] M. Takeoka, H. Takahashi, and M. Sasaki, Phys. Rev. A 77, 062315 (2008).

[28] P. Marek, H. Jeong, and M. S. Kim, Phys. Rev. A 78, 063811 (2008).

[29] C.-W. Lee, J. Lee, H. Nha, and H. Jeong, Phys. Rev. A 85, 063815 (2012).

[30] A. P. Lund, H. Jeong, T. C. Ralph, and M. S. Kim, Phys. Rev. A 70, 020101 (2004).

[31] M. Takeoka and M. Sasaki, Phys. Rev. A 75, 064302 (2007).

[32] J. B. Brask, I. Rigas, E. S. Polzik, U. L. Andersen, and A. S. Sørensen, Phys. Rev. Lett. 105, 160501 (2010).

[33] M. S. Kim, E. Park, P. L. Knight, and H. Jeong, Phys. Rev. A 71, 043805 (2005).

[34] R. Tualle-Brouri, A. Ourjoumtsev, A. Dantan, P. Grangier, M. Wubs, and A. S. Sørensen, Phys. Rev. A 80, 013806 (2009).

[35] S. Suzuki, M. Takeoka, M. Sasaki, U. L. Andersen, and F. Kannari, Phys. Rev. A 73, 042304 (2006).

[36] J. S. Neergaard-Nielsen et al., arXiv:1211.6634.

[37] R. Blandino, F. Ferreyrol, M. Barbieri, P. Grangier, and R. Tualle-Brouri, arXiv:1105.5510. 